\title{
Gastric duplication (bronchogenic) cyst mimicking a gastrointestinal stromal tumour
}

\author{
Sedat Belli¹, Turgut Noyan¹, Fazilet Kayaselçuk², Gürcan Erbay ${ }^{3}$
}

ABSTRACT

'Department of General Surgery, Başkent University Faculty of Medicine, Adana, Turkey

2Department of Pathology, Başkent University Faculty of Medicine, Adana, Turkey

${ }^{3}$ Department of Radiology, Başkent University Faculty of Medicine, Adana, Turkey

\section{Address for Correspondence Dr. Sedat Belli Department of General Surgery, Başkent University Faculty of Medicine, Adana, Turkey Phone.: +90322 3272727 e-mail: sedatbelli@hotmail.com}

Received: 07.06.2011 Accepted: 07.09.2011

@Copyright 2013 by Turkish Surgical Association Available online at www.ulusalcerrahidergisi.org
Gastric duplication cysts (GDC) lined with pseudostratified columnar ciliated epithelium (PCCE) are uncommon lesions that stem from a foregut developmental malformation. The clinical and radiological diagnosis is usually difficult. Definitive diagnosis can be established by histopathological examination. In this study, we report a rare case of a peri-gastric mass that was operated and reported as a bronchogenic cyst. Microscopically, the gastric cyst was lined by PCCE. Although rare, GDC should be included in the differential diagnosis of gastric wall masses. Surgical intervention is recommended in patients who have clinical symptoms.

Key Words: Gastric duplication cyst, bronchogenic cyst, foregut, pseudostratified columnar ciliated epithelium

\section{INTRODUCTION}

Bronchogenic cysts are rare benign congenital lesions (1-4). At any stage of the development of respiratory tract, these lesions result from abnormal budding of primitive tracheobronchial tube, two-thirds in the upper mediastinum, trachea, and around the bifurcation (1-4). However, outside the mediastinum and lungs, abdominal bronchogenic cysts are extremely rare (1-4). Despite being benign, recurrences can be observed in patients without complete resection (5). In our study, we aimed to present an atypically localized, rare bronchogenic cyst mimicking gastric stromal tumor.

\section{CASE PRESENTATION}

A 45-year-old female presented to our hospital with complaints of frequently recurring abdominal pain, weakness and fatigue. She was found to have lost five kilograms in the last month without a diet and was being followed up for elevated liver function tests for the last two years in another center. On physical examination, she had conjunctival pallor and abdominal fullness in the left upper quadrant. In routine laboratory examinations, mild hypochromic microcytic anemia and elevated liver function tests were detected. On endoscopy, she did not have any other pathology than gastritis. Thoraco-abdominal computed tomography (CT) scan revealed a well-defined, dense, cystic or semi-solid mass, $86 \times 71 \mathrm{~mm}$ in size, in the left subdiaphragmatic area, adjacent to the gastric cardia, that pushes the adrenal gland to the lateral and continue retro-crurally (Figure 1). CT-guided aspiration biopsy of the lesion was performed. The result showed mucoid material. The patient was planned for a laparotomy with a diagnosis of gastrointestinal stromal tumor (GIST). A midline incision was used to access the abdominal cavity. A cystic lesion, of $8 \times 7 \mathrm{~cm}$ in size was detected at the cardio-oesophageal junction, adjacent to the tail of the pancreas and spleen, behind the stomach, which was adherent to the diaphragm. A chest tube was inserted after injury to the diaphragm. The tube was withdrawn on the $3^{\text {rd }}$ postoperative day and the patient was allowed oral intake on the $4^{\text {th }}$ day. She was discharged on day 6 . Histopathological examination showed a single layered or pseudostratified columnar ciliated epithelium (PCCE) lining the cyst wall with dense foam cells and atrophic muscle fibers (Figure 2a, b). The epithelium did not show immunohistochemical staining with calretinin. Cytokeratin 7 (CK7) was diffuse positive in the epithelium, and CK20 was negative (Figure 2c). Epithelial staining with mucin and PAS was not observed. Based on these findings, the case was reported as a foregut duplication (bronchogenic) cyst.

\section{DISCUSSION}

Maier first reported bronchogenic cysts in 1948, which are extremely rare lesions (6). Bronchogenic cysts constitute $6.3 \%$ of primary mediastinal masses and $34 \%$ of primary mediastinal cysts (6). At any stage of the development of respiratory tract, these lesions result from abnormal budding of primitive tracheobronchial tube, and are generally localized in the mediastinum, and lung parenchyma (7). However, bronchogenic cysts may be found in atypical locations. As reported by Laraje et al. (4) the foregut gives rise to the pharynx, lower respiratory tract, oesophagus, stomach, duodenum, liver, gallbladder, pan- 
creas and bile duct system. During development of foregut, in the early embryonic period, anomalies such as duplication may be encountered. Therefore, duplication cysts can develop in any part of the digestive system from the mouth to anus $(2,4)$. Gastric duplications constitute $2-8 \%$ of all gastrointestinal duplications $(2,8)$.

Gastric duplication cysts (GDC) lined with pseudostratified columnar ciliated epithelium (PCCE) is a disease that manifests in advanced age (25-76 years) and does not show a clear gender predominance (10 females and 8 males) (1). It is usually asymptomatic (1-4). However, sometimes the patient presents

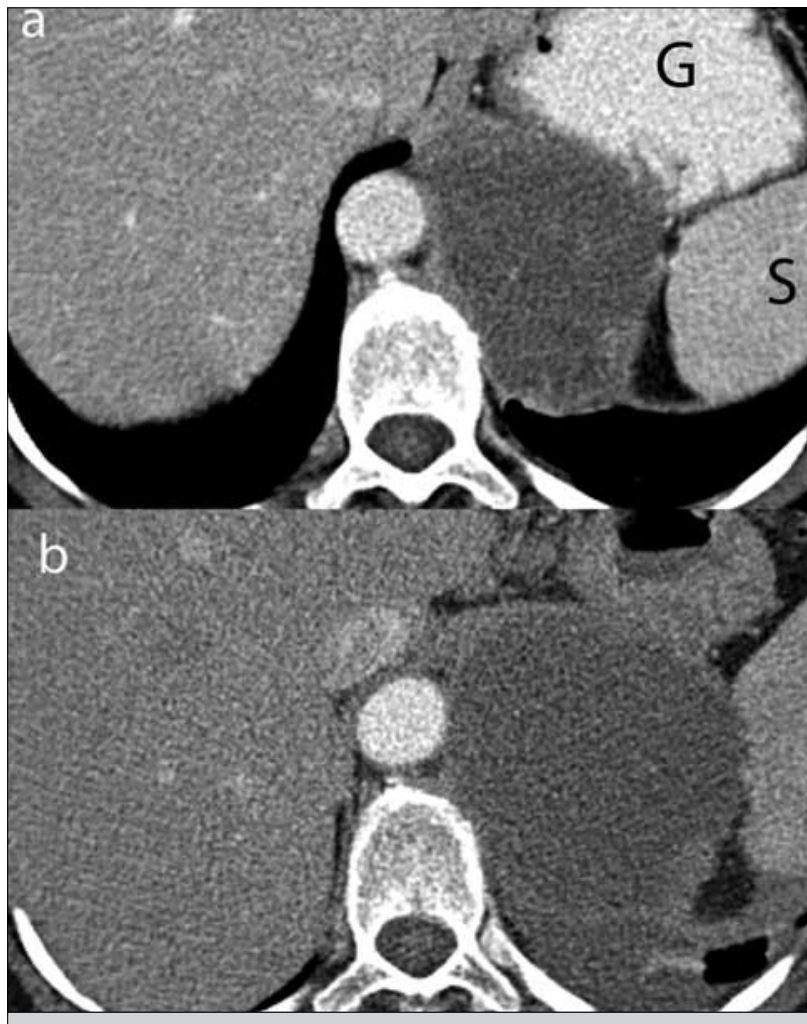

Figure 1. Axial CT images of the gastric bronchogenic cyst. a) subdiaphragmatic mass, adjacent to the gastric-cardiaspleen, with regular borders, and dense content in the precontrast images. b) weak contrast enhancement on the lesion wall in post-contrast images. $G$, stomach; $S$, spleen with complaints of abdominal pain, nausea, vomiting, weight loss and often non-specific symptoms such as epigastric mass (1-8). In a similar manner, our case first presented with nonspecific abdominal pain and weight loss. Other rare complications include gastric outlet obstruction, recurrent pancreatitis and hemoptysis (1-3). GDC is most commonly seen around the lesser curvature and gastro-oesophageal junction (1). In our case, the lesion was seen in a similar location, below the gastro-oesophageal junction. The literature review revealed the cyst size to vary from 1.7 to $15 \mathrm{~cm}$ (3). The cyst generally contains mucoid or gelatinous material (1-8).

The first step in the diagnosis of bronchogenic cyst is radiological examination. They appear as oval or round, hypodense homogeneous lesions with regular borders on CT (9). The cyst wall shows contrast enhancement and calcification (9). The cyst can be seen as a solid mass due to mucus accumulation $(3,9,10)$. In such cases, it is difficult to differentiate this lesion from other soft tissue masses. In our case, CT was extremely helpful regarding both the size and the location of the lesion. Nevertheless, CT was insufficient in defining the nature of the lesion, due to its mucoid structure. Bronchogenic cysts are often confused with a solid mass on CT scans $(3,9,10)$. GIST, oesophageal duplication cyst, retroperitoneal pseudomyxoma, cystic teratoma, bronchopulmonary sequestration, urothelial cyst of Mullerian origin and adrenal cystic tumors should be considered in the differential diagnosis $(3,11)$. In patients with difficulty in diagnosis, magnetic resonance imaging (MRI) or endoscopic ultrasonography can be used. It often appears hyperintense on T1-and T2-weighted MRI, due to dense proteinaceous content (9). In appropriate cases, preoperative CTguided needle biopsy may be helpful in differential diagnosis. The CT-guided needle biopsy of the lesion in our case revealed mucoid material. However, the sensitivity of needle biopsy in the diagnosis of GDC is low. Histopathological examination is required for definitive diagnosis.

In the majority of reported cases, duplication cysts are lined with gastrointestinal mucous membranes (1-3). However, GDC lined with PCCE is extremely rare. In a review published recently, Jiang et al. (1) reported on 18 cases. A wall that contains bronchial cartilage, smooth muscle, elastic tissue and mucous glands surrounds bronchogenic cysts containing mucoid ma-

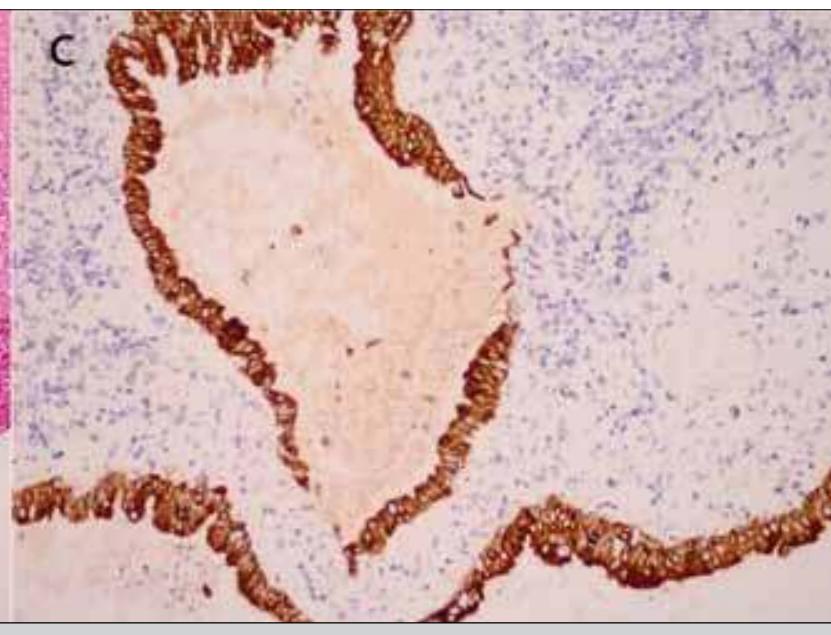

Figure 2. a) Cyst lined with pseudostratified columnar ciliated epithelium $(H+E, x 10)$. b) Close view of the lining epithelium $(H+E, x 20)$. c) strong positive staining in the epithelium with $C K 7(H+E, x 20)$ 
terial. The inner layer of this wall is covered by either ciliated columnar or cuboidal epithelium (1-3). In histopathological examination of the lesion in our case, the cyst was lined with PCCE and surrounded by smooth muscle fibers. Chondroid material was not observed.

Surgical resection is recommended in symptomatic patients $(1,2,10)$. Treatment options include open or laparoscopic resection and endoscopic snare resection (2). In our case, since the mass was large and attached to the left hemi-diaphragm a laparotomy was performed. Surgery is controversial in asymptomatic patients. The natural history of bronchogenic cysts and the percentage of asymptomatic patients who remain unnoticed throughout life are unknown. Although six patients with gastric cancer arising from gastric duplications have been reported recently, their true malignant potential is still unclear (12). Therefore, the inclination is to accept total excision of the cyst as the standard surgical approach. Recently, successful execution of CT-guided percutaneous or endoscopic needle aspiration have been reported, but because the cyst wall epithelium remains intact in these procedures, complications related to secretion accumulation may develop. Partial excision and these new drainage methods can relieve compression symptoms, but these methods do not eliminate the risk of infection, recurrence and malignant transformation (13).

The clinical conclusion in this case is the difficulty in preoperative or preoperative diagnosis, and even in postoperative macroscopic evaluation. This difficulty can be explained by its rare location in the stomach and its being a mass lesion with mucoid structure in radiologic evaluations. Due to non-specific clinical and radiological findings, GDC can easily be misdiagnosed as GIST.

\section{CONCLUSION}

Although abdominal bronchogenic cysts are extremely rare, they should be included in the differential diagnosis of gastric wall masses. In light of current knowledge, surgical intervention should be performed in symptomatic patients.

Peer-review: Externally peer-reviewed.
Author Contributions: Study concept and design - S.B., T.N., F.K.; Acquisition of data - S.B., T.N., F.K., G.E.; Analysis and interpretation of data - S.B., T.N., F.K., G.E.; Preparation of the manuscript - S.B., F.K., G.E.

Conflict of Interest: No conflict of interest was declared by the authors.

Financial Disclosure: The authors declared that this study has received no financial support.

\section{REFERENCES}

1. Jiang W, Zhang B, Fu YB, Wang JW, Gao SL, Zhang SZ, et al. Gastric duplication cyst lined by pseudostratified columnar ciliated epithelium: a case report and literature review. J Zhejiang Univ Sci B 2011; 12: 28-31. [CrossRef]

2. Wakabayashi H, Okano K, Yamamoto N, Suzuki Y, Inoue H, Kadota K, et al. Laparoscopically resected foregut duplication cyst (bronchogenic) of the stomach. Dig Dis Sci 2007; 52: 1767-70. [CrossRef]

3. Song SY, Noh JH, Lee SJ, Son HJ. Bronchogenic cyst of the stomach masquerading as benign stromal tumor. Pathol Int 2005; 55: 87-91. [CrossRef]

4. Laraja R, Rothenberg RE, Chapman J, Imran-UI-Haq, Sabatini MT. Foregut duplication cyst: a report of a case. Am Surg 1995; 61: 840-1.

5. Read CA, Moront M, Carangelo R, Holt RW, Richardson M. Recurrent bronchogenic cyst. An argument for complete surgical excision. Arch Surg 1991; 126: 1306-8. [CrossRef]

6. Maier HC. Bronchogenic cyst of the mediastinum. Ann Surg 1948; 127: 476-502. [CrossRef]

7. Di Lorenzo M, Collin PP, Vaillancourt R, Duranceau A. Bronchogenic cyst. J Pediatr Surg 1989; 24: 988-91. [CrossRef]

8. Kim DH, Kim JS, Nam ES, Shin HS. Foregut duplication cyst of the stomach. Pathol Int 2000; 50: 142-5. [CrossRef]

9. Chung JM, Jung MJ, Lee W, Choi S. Retroperitoneal bronchogenic cyst presenting as adrenal tumor in adult successfully treated with retroperitoneal laparoscopic surgery. Urology 2009; 73: 13-5. [CrossRef]

10. Vos CG, Hartemink KJ, Golding RP, Oosterhuis JW, Paul MA. Bronchogenic cysts in adults: frequently mistaken for a solid mass on computed tomography. Wien Klin Wochenschr 2011; 123: 179-82. [CrossRef]

11. Haddadin WJ, Reid R, Jindal RM. A retroperitoneal bronchogenic cyst: a rare cause of a mass in the adrenal region. J Clin Pathol 2001; 54: 801-2. [CrossRef]

12. Kuraoka K, Nakayama H, Kagawa T, Ichikawa T, Yasui W. Adenocarcinoma arising from a gastric duplication cyst with invasion to the stomach: a case report with literature review. J Clin Pathol 2004; 57: 428-31. [CrossRef]

13. Rice DC, Putnam JB Jr. Recurrent bronchogenic cyst causing recurrent laryngeal nerve palsy. Eur J Cardiothorac Surg 2002; 21: 561-3. [CrossRef] 